\title{
Dynamics of Alfvén waves in the night-side ionospheric Alfvén resonator at mid-latitudes
}

\author{
V. V. Alpatov ${ }^{1}$, M. G. Deminov ${ }^{2}$, D. S. Faermark ${ }^{1}$, I. A. Grebnev ${ }^{1}$, and M. J. Kosch ${ }^{3}$ \\ ${ }^{1}$ Institute of Applied Geophysics, Rostokinskaya st., 9, Moscow, 129128, Russia \\ ${ }^{2}$ Institute of Terrestrial Magnetism, Ionosphere and Radio Wave Propagation, RAS, Troitsk, Moscow region, 142190, Russia \\ ${ }^{3}$ Department of Communication Systems, Lancaster University, LA1 4YR Lancaster, United Kingdom
}

Received: 11 October 2002 - Revised: 28 September 2004 - Accepted: 9 November 2004 - Published: 28 February 2005

\begin{abstract}
A numerical solution of the problem on dynamics of shear-mode Alfvén waves in the ionospheric Alfvén resonator (IAR) region at middle latitudes at nighttime is presented for a case when a source emits a single pulse of duration $\tau$ into the resonator region. It is obtained that a part of the pulse energy is trapped by the IAR. As a result, there occur Alfvén waves trapped by the resonator which are being damped. It is established that the amplitude of the trapped waves depends essentially on the emitted pulse duration $\tau$ and it is maximum at $\tau=(3 / 4) T$, where $T$ is the IAR fundamental period. The maximum amplitude of these waves does not exceed $30 \%$ of the initial pulse even under optimum conditions. Relatively low efficiency of trapping the shear-mode Alfvén waves is caused by a difference between the optimum duration of the pulse and the fundamental period of the resonator. The period of oscillations of the trapped waves is approximately equal to $T$, irrespective of the pulse duration $\tau$. The characteristic time of damping of the trapped waves $\tau_{\mathrm{dec}}$ is proportional to $T$, therefore the resonator Q-factor for such waves is independent of $T$. For a periodic source the amplitude-frequency characteristic of the IAR has a local minimum at the frequency $\pi / \omega=(3 / 4) T$, and the waves of such frequency do not accumulate energy in the resonator region. At the fundamental frequency $\omega=2 \pi / T$ the amplitude of the waves coming from the periodic source can be amplified in the resonator region by more than $50 \%$. This alone is a basic difference between efficiencies of pulse and periodic sources of Alfvén waves. Explicit dependences of the IAR characteristics $\left(T, \tau_{\mathrm{dec}}\right.$, Q-factor and eigenfrequencies) on the altitudinal distribution of Alfvén velocity are presented which are analytical approximations of numerical results.
\end{abstract}

Key words. Ionosphere (Mid-latitude ionosphere, Wave propagation, Modeling and forecasting) - Magnetospheric physics (MHD waves and instabilities, Magnetosphereionosphere interactions)

Correspondence to: D. S. Faermark

(mostfaer@mtu-net.ru)

\section{Introduction}

Shear-mode Alfvén waves in the ionosphere in the frequency range 0.1 to $10 \mathrm{~Hz}$ can be reflected from regions above and below the F2-layer maximum because of large (relative to the wave length in the medium) gradients of the refractive index (Polyakov and Rapoport, 1981; Belyaev et al., 1990). The occurrence of such reflections and the absence of a group velocity transverse to the geomagnetic field implies that the resonator for shear-mode Alfvén waves - the ionospheric Alfvén resonator (IAR) - should exist (Polyakov and Rapoport, 1981).

The IAR has been identified in the ground-based observations both at middle (Belyaev et al., 1990, 2000) and high (Belyaev et al., 1999) latitudes. Analysis of Freja satellite data (e.g. Grzesiak, 2000) also confirms the existence of this phenomenon in the topside ionosphere. The IAR properties have been studied theoretically by Polyakov and Rapoport (1981); Trakhtengertz and Feldstein (1984, 1991); Lysak (1986, 1988, 1991); Belyaev et al. (1990); Demekhov et al. (2000); Pokhotelov et al. (2000, 2001) and Prikner et al. (2000).

The first numerical model that fully resolved the vertical ionospheric structure has been presented by Lysak (1997, 1999). While previous models studied the response of the ionosphere to waves at a fixed frequency, this model was the first that allowed one to investigate the effects in the ionosphere under action of a field-aligned current pulse with arbitrary time dependence generated by a source of this current in the magnetosphere.

In this paper a numerical model of similar type is used but with another position of the source and a number of simplifications specified by the statement of the problem. The main objective of this work is to analyse features of trapping by the ionospheric resonator of a single pulse of a shear-mode Alfvén wave for the case when the energy losses of the wave at the E-layer heights are insignificant. This corresponds to night-time conditions at middle latitudes which according to observation data, the IAR Q-factor is relatively high (Belyaev et al., 2000). The E-layer is optically thin at frequencies 
lower than 5 to $10 \mathrm{~Hz}$ for these geophysical conditions, and it allows us to use integrated conductivity of the ionosphere. Besides, low losses of energy of shear-mode Alfvén waves at the E-layer heights allow us to neglect the connection of these waves with isotropic fast compressional waves. This connection was the focus of Lysak's $(1997,1999)$ results. Therefore, Lysak's $(1997,1999)$ model is two-dimensional while our model is one-dimensional. Note that a single pulse of Alfvén wave is generated at an artificial action (chemical release experiment or rocket exhaust gases) in the ionosphere (see, e.g. Marklund et al., 1987; Gaidukov et al., 1993; Deminov et al., 2001). Therefore, we put a source of Alfvén waves inside the resonator, more precisely, at the heights close to the upper border of the E-layer.

The other purpose of this work is to compare characteristics of the Alfvén waves generated by a pulse source and trapped by the resonator with characteristics of the Alfvén waves generated by a periodic source and retained by the resonator. Therefore, two versions of the Alfvén wave generators - a single pulse and a periodic wave - will be considered below.

\section{Formulation of the problem}

Propagation of magnetohydrodynamic waves in three regions is taken into account: in the upper ionosphere at the heights $h \geq h_{0} \approx 150 \mathrm{~km}$, where the plasma is magnetized and inertial currents are important; in the $\mathrm{E}$ layer of the ionosphere $\left(h_{0} \geq h \geq h_{B} \approx 100 \mathrm{~km}\right)$, where ionospheric conductivity currents are important; and in the electrically neutral atmosphere between $h_{B}$ and the highly conducting surface of the Earth, where there are no charges and currents if the displacement current is neglected (see, e.g. Nishida, 1978).

It is assumed that background electric fields and currents are absent, i.e. the high-latitudes conditions are not considered. We shall consider only the shear-mode Alfvén wave, i.e. the effects of transformation of this wave into the isotropic fast compressional wave will be neglected. Therefore, in what follows for brevity the shear-mode Alfvén wave will be called the Alfvén wave or simply the wave. The system of equations for Alfvén waves in the upper mid-latitude ionosphere is

$1 /\left(\mu_{0} V_{A}^{2}\right) \cdot \partial / \partial t \nabla_{\perp} \cdot \boldsymbol{E}=-\partial J_{z} / \partial z$,

$\mu_{0} \partial J_{z} / \partial t=-\partial / \partial z \nabla_{\perp} \cdot \boldsymbol{E}$,

where the $z$ axis is directed upwards opposite to the geomagnetic field $\left(\mathbf{e}_{z}=-\boldsymbol{B}_{0} / B_{0}\right), J_{z}$ and $\nabla_{\perp} \cdot \boldsymbol{E}$ are the fieldaligned current and divergence of the wave electric field, respectively, the field $\boldsymbol{E}$ is orthogonal to $\boldsymbol{B}_{0}, V_{A}$ is the Alfvén velocity, and $\mu_{0}$ is the magnetic permeability of free space. The first equation is deduced from the current continuity condition and from Ohm's law for collisionless plasma $\boldsymbol{J}_{\perp}=1 /\left(\mu_{0} V_{A}^{2}\right) \partial \boldsymbol{E} / \partial t$ (see, e.g. Nishida, 1978). The second equation is the consequence of Faraday's and Amper's laws. Note that Eqs. (1) and (2) coincide with the equations of Lysak's detailed model (1997, 1999), if in Lysak's equations one neglects collisions, assumes that the cross scale of the wave is not too small, and takes into account that for the Alfvén wave the following condition is valid:

$\nabla_{\perp} \times \boldsymbol{E}=0$.

The continuity equation for the current can be written as

$\partial J_{z} / \partial z+\nabla_{\perp} \cdot\left(\boldsymbol{J}_{\perp}+\boldsymbol{J}_{\perp}^{\mathrm{ext}}\right)=0$,

where $\boldsymbol{J}_{\perp}^{\text {ext }}$ is the transverse component of extrinsic current which is considered as a source of waves. Taking into account Eq. (3), the continuity equation in the conducting layer can be written as

$\partial J_{z} / \partial z+\nabla_{\perp} \cdot\left(\sigma_{p} \boldsymbol{E}+\boldsymbol{J}_{\perp}^{\mathrm{ext}}\right)=0$,

where $\sigma_{p}$ is the Pedersen conductivity of the ionosphere. For definiteness, we place the source inside the conducting layer. In Sect. 5 we shall return to this question and show that our conclusions basically do not depend of the source localization. The conducting layer was assumed to be optically thin, and Eq. (5) was integrated over $z$ from the bottom up to the top border of the conducting layer. Taking into account that at the bottom border of the conducting layer there are no currents, we obtain the boundary condition for the system of Eqs. (1) and (2) at the height $h=h_{0}=150 \mathrm{~km}$ :

$J_{z}\left(h_{0}=150 \mathrm{~km}\right)=\left(-\Sigma_{p} \nabla_{\perp} \cdot \boldsymbol{E}-\nabla_{\perp} \cdot \int \boldsymbol{J}_{\perp}^{\mathrm{ext}} d z\right) / \sin I$,

where $\Sigma_{P}$ is the height-integrated Pedersen conductivity of the ionosphere and $I$ is the magnetic inclination. The boundary condition (Eq. 6) is the condition of short circuit of the source current by conductivity currents and the field-aligned current of the wave. It allows us not to consider propagation of the waves at the region below the E-layer.

The processes inside the source are not studied here, therefore, we put

$-\nabla_{\perp} \cdot \int \boldsymbol{J}_{\perp}^{\mathrm{ext}} d z=J_{0} F(t)$,

where $|F(t)| \leq 1$. For this case, the wave is radiated into the region $h>h_{0}$, and $J_{0}$ is the amplitude of the field-aligned current of this wave close to the source, if energy losses of this wave in the E-layer are neglected.

The upper boundary $h_{1}$ for Eqs. (1) and (2) is located above the IAR. At this height the condition for radiation of waves along the geomagnetic field into the magnetosphere is specified and the subsequent reflection of waves from the conjugate ionosphere is ignored.

The system of Eqs. (1) and (2) was solved numerically by the method of characteristics (see, e.g. Rozhdestvensky and Yanenko, 1978) in the following variables (Riemannian invariants):

$p=\nabla_{\perp} \cdot \boldsymbol{E}+R J_{z}, q=\nabla_{\perp} \cdot \boldsymbol{E}-R J_{z}$,

where $R=\mu_{0} V_{A}=1 / \Sigma_{A}$, and $\Sigma_{A}$ is the wave conductivity. Using these variables, the system of Eqs. (1) and (2) takes the characteristic form

$\left(1 / V_{A}\right) \partial p / \partial t+\partial p / \partial z=D, \quad\left(1 / V_{A}\right) \partial q / \partial t-\partial q / \partial z=D$, 
where $D=0.5(p-q) \partial \ln \left(V_{A}\right) / \partial z$. Boundary conditions for Eq. (8), taking into account Eq. (6), are

$p\left[1+\Sigma_{P} /\left(\Sigma_{A} \sin I\right)\right]-$ $q\left[1-\Sigma_{P} /\left(\Sigma_{A} \sin I\right)\right]=2 J_{0} F(t) / \Sigma_{A}$ for $h=h_{0}$,

$q=0$

for $h=h_{1}$.

From Eq. (8) it is seen that the wave $p$ propagates only upwards, and $q$ only downwards. The boundary condition (Eq. 9b) is the condition for radiation of the whole wave upwards into the magnetosphere at the upper boundary, so that no part of this wave is reflected downwards. Equations (7) to (9) are the formulation of the problem. Note that the waves $p$ and $q$ are always present simultaneously in a non-uniform medium $\left(\partial \ln \left(V_{A}\right) / \partial z \neq 0\right)$, and the term $D$ in Eq. (8) describes the redistribution of energy between these waves. In this formulation of the problem the direction of Pointing vector of the Alfvén wave coincides with $J_{z}$. Therefore in what follows the main attention will be given to the dynamics of the field-aligned currents in the IAR region.

\section{Geophysical conditions}

From ground-based observations at middle latitudes it is known that the IAR Q-factor is maximum at night (Belyaev et al., 2000). Figure 1a shows the altitude distribution (along the geomagnetic field) of the Alfvén wave refractive index $n_{A}=c / V_{A}$ obtained using the IRI model (Bilitza, 1997) for medium solar activity - a relative sunspot number $W=70$, middle latitudes - an $L$-shell equal to 1.8 , in February, at midnight.

The analytical approximation of $n_{A}$ altitude distribution for the F2-layer, where $\mathrm{O}^{+}$ions dominate, is

$n_{A}=n_{A m} \exp (0.25(1-x-\exp (-x))), \quad x=4\left(h-h_{m}\right) / L$,

where $h_{m}$ is the height of $n_{A}$ maximum for which $n_{A}=n_{A m}=c / V_{A m}$ and $L$ is the characteristic scale of the $n_{A}$ change with height for $h>h_{m}$. In the case under consideration $n_{A m}=407, h_{m}=345 \mathrm{~km}$, and $L=240 \mathrm{~km}$. Note that qualitatively the relation $L=L\left(\mathrm{O}^{+}\right)=2 k\left(T_{e}+T_{i}\right) / m\left(\mathrm{O}^{+}\right) g$ is valid for the F2-layer in the region above the layer maximum. Above the F2-layer, in the protonosphere, where $\mathrm{H}^{+}$ becomes the dominant ion, the characteristic scale of $n_{A}$ increases with height. At the heights where protons dominate $\left(N\left(\mathrm{H}^{+}\right) \gg \mathrm{N}\left(\mathrm{O}^{+}\right)\right)$the scale is given by $1 / L=1 / L\left(\mathrm{H}^{+}\right)$ $=0.5 m\left(\mathrm{H}^{+}\right) g / k\left(T_{e}+T_{i}\right)+\partial \ln \left(B_{0}\right) / \partial z$, where $B_{0}$ is the geomagnetic field and $\partial \ln \left(B_{0}\right) / \partial z \approx-3 /\left(R_{E}+h\right)$. The height where $1 / L\left(\mathrm{H}^{+}\right)=0$ corresponds to $n_{A}$ minimum. In our case this $n_{A}$ minimum is located close to $1300 \mathrm{~km}$, and the protonosphere is located higher than $700 \mathrm{~km}$.

In what follows, the region near the $n_{A}$ maximum is called the central part of the IAR. The upper and lower walls of the IAR are located above and below the central part of the IAR where $n_{A}$ decreases with distance from this central region. In the considered case the lower wall of the IAR is very steep and extends to the upper boundary of the E-layer at $150 \mathrm{~km}$.

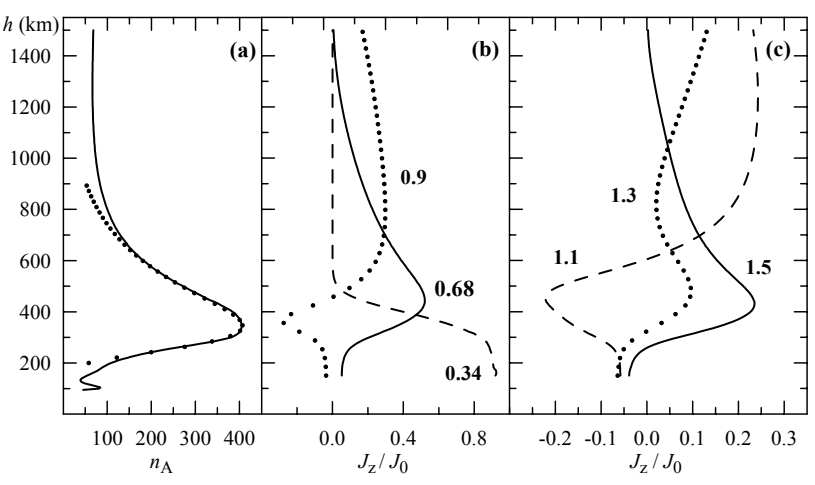

Fig. 1. Dependence on height $h$ of: (a) the Alfvén wave refractive index $n_{A}$, (dots are the analytical approximation of $n_{A}$ according to Eq. (10); (b), (c) relative values of the field-aligned current of the Alfvén waves $J_{z} / J_{0}$ at fixed points in time. Numerals at the curves are times $t$ in seconds from the turn on of the pulse source. The duration of the pulse is $\tau=\pi / \omega=0.68 \mathrm{~s}$.

The upper wall is less steep and extends up to $\approx 1300 \mathrm{~km}$ where $n_{A}=n_{\text {Amin }}=66$. In this case the condition (Eq. $9 \mathrm{~b}$ ) for radiation of the wave into the magnetosphere is specified at the height $h=h_{1}=1500 \mathrm{~km}$, i.e. higher than the IAR.

By analogy with analytical studies of the resonator (Polyakov and Rapoport, 1981; Lysak, 1991) we designate the depth of the resonator as $\varepsilon$ :

$\varepsilon=n_{\mathrm{Amin}} / n_{A m}$.

In the considered case $\varepsilon=0.16$.

In Eq. (6) it was taken that the conducting E-layer is optically thin for the Alfvén wave of frequency $\omega$. This condition is valid if (see, e.g. Lysak, 1999):

$\delta=\left(2 / \mu_{0} \omega \sigma_{P}\right)^{1 / 2} \gg L_{P}$,

where $\delta$ is the skin-depth, $L_{P}$ is the width of the conductive layer of the ionosphere, $\Sigma_{P}=L_{P} \sigma_{P}$. In this case $\sigma_{P}=2 \cdot 10^{-6}$ $\mathrm{mho} /, \Sigma_{P}=0.05 \mathrm{mho}, L_{P}=25 \mathrm{~km}$, and the conductive layer is optically thin for frequencies $f=\omega / 2 \pi \ll 100 \mathrm{~Hz}$. Hence, for the analyzed range of frequencies 0.1 to $10 \mathrm{~Hz}$ the condition is valid so long as the conductive layer of the ionosphere is thin.

Note one of the consequences of Eq. (10). For this purpose we take into account that at middle latitudes the change in height by dh corresponds to a distance along the geomagnetic field $d s=d h / \sin I$. If one accepts that Eq. (10) is valid at all heights and sinI does not depend on height, then integration of $2\left(n_{A} / c\right)$ along the geomagnetic field from the Earth's surface up to infinity gives the period of movement of the Alfvén wave between these boundaries (bounce period):

$T_{\text {bmax }}=(1 / \sqrt{2}) \exp (1 / 4) \Gamma(1 / 4)(L / \sin I) / V_{A m}$

$=3.29(L / \sin I) / V_{A m}$,

where $\Gamma(1 / 4)$ is a Gamma function. In this case $T_{\mathrm{bmax}}=1.2 \mathrm{~s}$. This value of $T_{\text {bmax }}$ remains constant if the bottom border of 


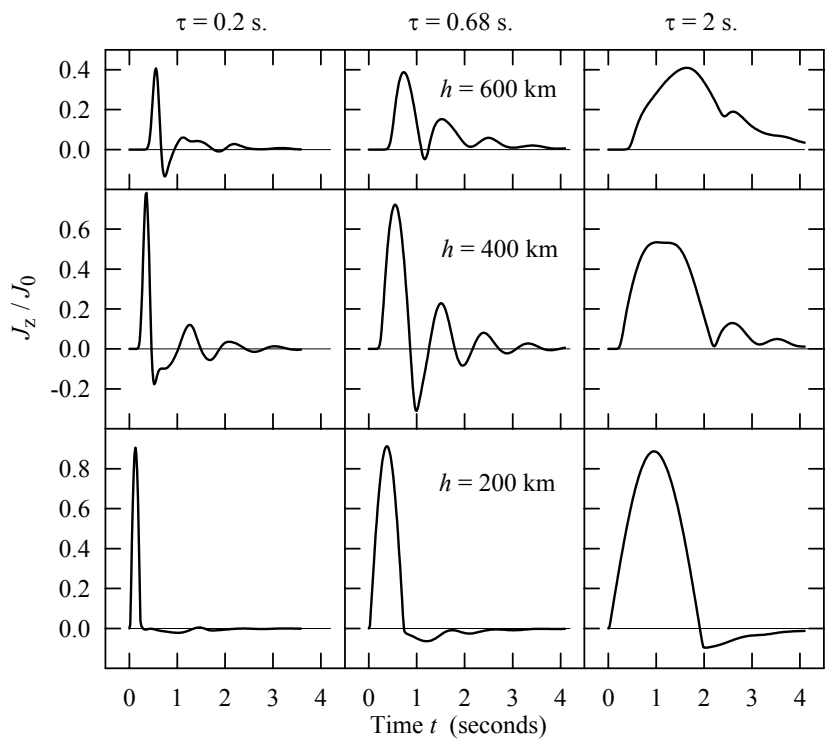

Fig. 2. Dependence on time $t$ of relative values of the Alfvén wave field-aligned current $J_{z} / J_{0}$ at three fixed heights $h$ for the emitted pulse durations $\tau=0.2,0.68$, and $2 \mathrm{~s}$. Time is measured from turn on of the pulse source.

integration is located at the height $h_{0}=150 \mathrm{~km}$. Integration between the bottom and top borders of the F2-layer (in this case from 150 up to $700 \mathrm{~km}$ ) gives $T_{b}=1 \mathrm{~s}$. It is seen that the $T_{b}$ value does not strongly differ from $T_{\mathrm{bmax}}$ and the difference between them is near $20 \%$. It allows us to assume that $T_{b}$ dependence on the ionosphere parameters is similar to above $T_{\text {bmax }}$ dependence on these parameters:

$$
T_{b}=2.7(L / \sin I) / V_{A m}, \Omega_{b}=2 \pi / T_{b}=2.3\left(V_{A m} / L\right) \sin I .
$$

Equation (12) can be considered as analytical approximation of numerical solution of this problem which is applicable for the whole really observed range of $n_{A m}, L$ and $h_{m}$ changes in Eq. (10). Comparison of $T_{\mathrm{bmax}}$ and $T_{b}$ shows that the inaccuracy of such approximation is smaller than $20 \%$. This property of Eq. (10) is used below for the approximation of the IAR eigenfrequencies.

\section{Single pulse of the Alfvén wave}

Let the function $F(t)$ in the boundary condition (9a) be

$F(t)=\sin (\omega t)$ for $0 \leq t \leq \pi / \omega ; \quad F(t)=0$ for $t>\pi / \omega$,

i.e. the source radiates upwards (into the region $h>h_{0}$ ) an Alfvén wave as a pulse of duration $\tau=\pi / \omega$. The field-aligned current amplitude of this wave near the source is equal to $J_{0}$ if one neglects the Alfvén wave energy losses into the E-layer. Below we consider relative changes in the field-aligned current of the Alfvén waves $J_{z} / J_{0}$ in the IAR region.

We put frequency $\omega=4.6 \mathrm{~s}^{-1}(f \approx 0.7 \mathrm{~Hz})$ which corresponds to the pulse duration of the emitted Alfvén wave $\tau=0.68 \mathrm{~s}$. Figures $1 \mathrm{~b}$ and $\mathrm{c}$ show the altitude distribution of the field-aligned current of the Alfvén waves $J_{z} / J_{0}$ for fixed times $t$ from the moment the source was switched on. At $t=0.34 \mathrm{~s}$ the generated pulse is maximum near the source. At this time just above the source $(h \approx 150 \mathrm{~km})$ the field-aligned current is $J_{z} / J_{0}=0.925$, i.e. the energy losses in the E-layer do not exceed 7 to $8 \%$ (see Eq. 9a), and reflection of the waves from the IAR lower wall is almost specular. By the time the source is switched off ( $t=0.68 \mathrm{~s}$ ) the pulse maximum reaches the IAR upper wall. At this time $J_{z}>0$ in the whole IAR region. At further propagation of the pulse some part reflects from the IAR upper wall and then reflects from the lower wall. As a result, a region is formed where $J_{z}<0$. At $t=0.9 \mathrm{~s}$ this region is located below approximately $450 \mathrm{~km}$. At $t=1.1 \mathrm{~s}$ a very wide maximum of the pulse reaches the IAR upper wall. By this time $J_{z}<0$ in the whole region of the F2-layer $(200 \leq h \leq 600 \mathrm{~km})$ and $J_{z}$ minimum is located in the central part of this region. This extremum is formed by $p$ and $q$ waves in which $J_{z}<0$ (see Eq. 7). The $q$ wave is the wave reflected from the upper wall of the resonator and after that it reaches $J_{z}$ minimum. The $p$ wave is associated with the $q$ waves from previous times where it reached the lower wall of the resonator, then reflected from this wall and reached $J_{z}$ minimum. At $t>1.1 \mathrm{~s}$ the $p$ and $q$ waves continue their movement and, having reflected from the resonator walls, reach the central part of the resonator again. They form $J_{z}$ maximum, as at this time $J_{z}>0$ in the $p$ and $q$ waves due to their reflections. In Fig. 1 this maximum is seen at $t=1.5 \mathrm{~s}$. These are just the oscillations of the Alfvén waves between the resonator upper and lower walls, i.e. the waves trapped by the resonator. The frequency of these oscillations is $\Omega \approx 6.9 \mathrm{~s}^{-1}$.

It was noted above that reflections of the waves from the IAR lower wall are almost specular and the energy losses in the E-layer are insignificant. The energy losses through radiation to the magnetosphere are important. This is evident from the sharp decrease in $J_{z} / J_{0}$ at the heights $h>800 \mathrm{~km}$ at transition from $t=1.1$ to $t=1.3 \mathrm{~s}$ when the main maximum of the Alfvén wave pulse crosses the IAR upper boundary. Therefore, most of the energy of the waves trapped by the resonator is concentrated close to the central part of the resonator, i.e. at the heights of the F2-layer. This is evident from Fig 2, where $J_{z} / J_{0}$ dependences on time at three fixed heights are shown for different durations of the emitted pulse of the Alfvén wave. Secondary maxima of $J_{z} / J_{0}$ are most distinct near the IAR central part at the height $h=400 \mathrm{~km}$, but they are much weaker at $h=600 \mathrm{~km}$ and practically absent at $h=200 \mathrm{~km}$. At the height of $400 \mathrm{~km}$ the intervals between maxima, beginning with the second maximum (for a short pulse, with the third one), almost do not depend on the emitted pulse duration: $T=0.91 \pm 0.04 \mathrm{~s}$. The value $T \approx 0.91 \mathrm{~s}$ is the fundamental period of the IAR which corresponds to the fundamental frequency of the resonator $\Omega \approx 6.9 \mathrm{~s}^{-1}$.

The frequency $\Omega$ is determined by internal properties of the resonator, i.e. depends only on the altitude distribution of the Alfvén wave refractive index $n_{A}$ in the resonator region. An approximation of this dependence, taking into account 
Eq. (10), is

$\Omega \approx 2.6\left(c / n_{A m} L\right) \sin I \approx 2.6\left(V_{A m} / L\right) \sin I$.

The fact that most of the wave energy trapped by the resonator is concentrated at the F2-layer heights was taken into account in deducing Eq. (14). Therefore, $\Omega$ dependence on the ionosphere parameters is similar to the one for $\Omega_{b}$ (see Eq. 12). Calculations show that for night hours at middle latitudes Eq. (14) is valid for any level of solar activity and for any season with a deviation of less than 15 to $20 \%$.

If approximation (Eq. 10) is used, the IAR fundamental period corresponds approximately to the double time of the Alfvén wave propagation from 200 up to $600-650 \mathrm{~km}$. Hence, almost the whole F2 region participates in the formation of the trapped waves. Height $h^{*} \approx h_{m}+L / 4 \approx 400 \mathrm{~km}$ corresponds to the half time of the wave propagation at the distance from 200 to $650 \mathrm{~km}$. This height corresponds to the area of peak values of $J_{z} / J_{0}$ in the trapped waves, as superposition of the waves reflected from the top and bottom walls of the resonator occurs in this area (see Figs. 1 and 2). The distance from $200 \mathrm{~km}$ up to $h_{m}$ is half the interval from $h_{m}$ up to $650 \mathrm{~km}$, and for qualitative estimations one can take the characteristic scale of $n_{A}$ change in the region of the resonator bottom wall to be equal to $0.5 \mathrm{~L}$.

The waves trapped by the resonator are damped out (see Fig. 2). Their characteristic damping time $\tau_{\mathrm{dec}}$ can be found from the ratio of $J_{z} / J_{0}$ maxima in the central part of the resonator, beginning with the second maximum, i.e. the ratio of the second maximum to the third one, etc. In the considered case $\tau_{\text {dec }} / T \approx 0.95 \pm 0.1$ irrespective of the emitted pulse duration. Additional calculations at fixed $\mathrm{T}$ show that $\tau_{\mathrm{dec}} \sim 1 / \varepsilon$ for the case when energy losses of the waves in the E-layer can be neglected. Similar dependence of $\tau_{\mathrm{dec}}$ on $\varepsilon$ also follows from the analytical solution of the problem for a periodic source (Belyaev et al., 1990). Therefore, approximately

$\tau_{\mathrm{dec}}=(0.15 / \varepsilon) T=(0.15 / \varepsilon) 2 \pi / \Omega$.

Calculations for mid-latitude winter conditions at nearmidnight hours at low, middle and high solar activity show that the inaccuracy of approximation (Eq. 15) does not exceed 10 to $15 \%$. Nevertheless, it should be noted once more that this approximation is applicable only for a case when energy losses of the waves in the E-layer can be neglected.

The resonator Q-factor is usually calculated as a ratio of the real part of the resonator eigenfrequency $\omega^{*}$ to its imaginary part: $Q=0.5 \operatorname{Re}\left(\omega^{*}\right) / \operatorname{Im}\left(\omega^{*}\right)$. In the considered case $\operatorname{Re}\left(\omega^{*}\right)=\Omega, \operatorname{Im}\left(\omega^{*}\right)=1 / \tau_{\mathrm{dec}}$ for the trapped waves, and it follows from Eq. (15) that the IAR Q-factor for these waves is

$Q \approx 0.5 / \varepsilon$.

For the reasons noted above this equation is valid only for a version when energy losses of the waves in the E layer can be neglected.

The amplitude of the trapped waves $\left(J_{z} / J_{0}\right)_{\max }$ depends on the duration of the emitted pulse $\pi / \omega$. Here $\left(J_{z} / J_{0}\right)_{\max }$ has its

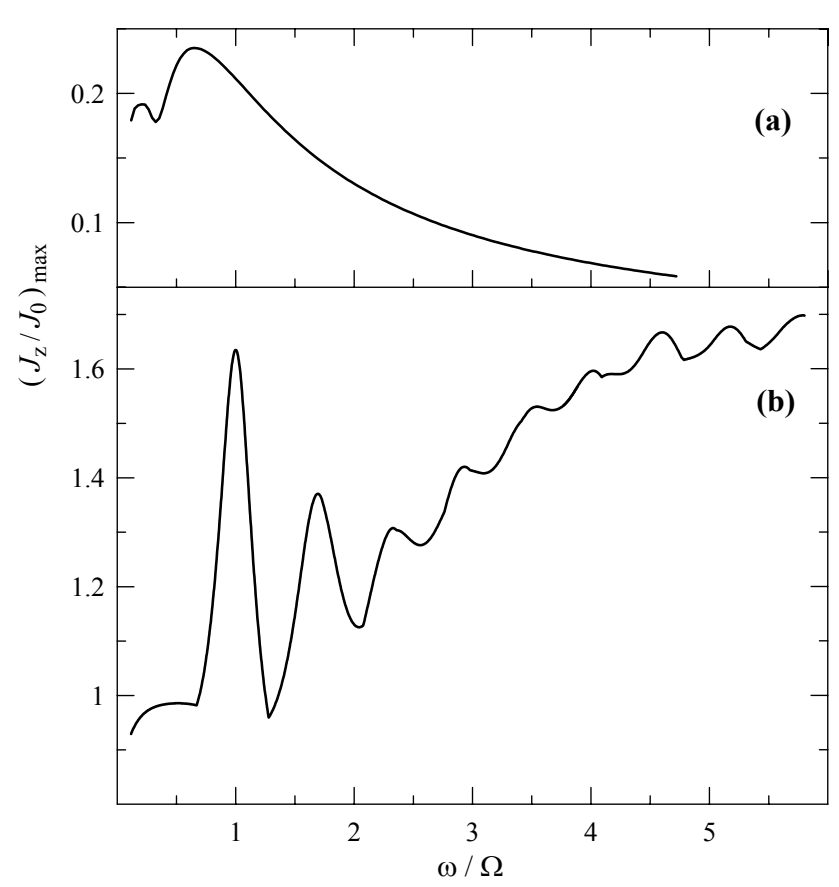

Fig. 3. Amplitude-frequency characteristics of the resonator for a single pulse (a) and a periodic wave after a stationary state has been attained (b). $\left(J_{z} / J_{0}\right)_{\max }$ is the maximum of the relative value of the Alfvén wave field-aligned current in the resonator region, $\omega$ is the wave frequency, $\Omega$ is the IAR fundamental frequency.

greatest value at the second maximum of $J_{z} / J_{0}$ in the height interval 200 to $650 \mathrm{~km}$. This amplitude is maximum when

$\omega=\omega_{R} \approx \Omega / 1.5$.

The frequency $\omega_{R}$ corresponds to the pulse duration $\tau_{R}=\pi / \omega_{R}$, and from Eq. (17) it follows that the amplitude of the waves trapped by the resonator is maximaum at the emitted pulse duration

$\tau=\tau_{R} \approx(3 / 4) T$.

We name $\omega_{R}$ as the optimum frequency of the emitted pulse. It is seen that this frequency is smaller than the resonator fundamental frequency $\Omega$. It is caused by the fact that $\left(J_{z} / J_{0}\right)_{\max }$ is formed as a result of superposition of the waves reflected from the resonator bottom and top walls which have been formed by one pulse of the wave emitted upwards. The wave reflected from the resonator bottom wall passes twice to the IAR central part, more precisely, to the height $h^{*} \approx 400 \mathrm{~km}$ : it is reflected from the lower part of the top wall and then is reflected once more from the bottom wall. The wave reflected from the upper part of the resonator top wall passes once to $h^{*}$. The numerical coefficient 1.5 in Eq. (17) corresponds to optimum conditions when the times of passage of these waves are in agreement between themselves. Note that the characteristics of the Alfvén wave shown in Fig. 1 correspond to the optimum duration of the emitted pulse $\tau=\tau_{R}=\pi / \omega_{R}=0.68 \mathrm{~s}$. 
Table 1. Parameters of maxima at the amplitude-frequency characteristic of the resonator for a periodic source obtained from the numerical solution of the problem and from approximation of the resonant frequencies by Eq. (19) $\left(\omega_{n} / \Omega\right)_{a p}$.

\begin{tabular}{cccccccccc}
\hline$N$ & 1 & 2 & 3 & 4 & 5 & 6 & 7 & 8 & 9 \\
\hline$\omega_{n} / \Omega$ & 1.00 & 1.69 & 2.33 & 2.94 & 3.55 & 4.06 & 4.61 & 5.19 & 5.79 \\
$\left(J_{z} / J_{0}\right)_{\max }$ & 1.63 & 1.37 & 1.31 & 1.42 & 1.53 & 1.60 & 1.67 & 1.68 & 1.70 \\
$\left(\omega_{n} / \Omega\right)_{a p}$ & 1.00 & 1.66 & 2.30 & 2.92 & 3.52 & 4.11 & 4.68 & 5.23 & 5.76 \\
\hline
\end{tabular}

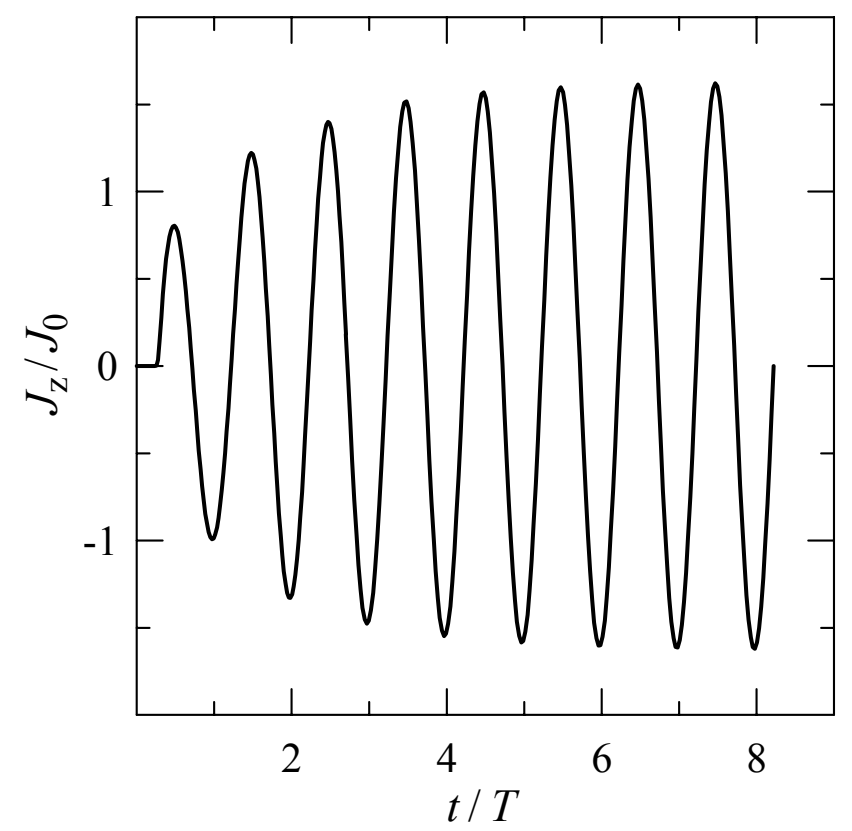

Fig. 4. Dependence of $J_{z} / J_{0}$ on time $t$ at the fixed height $400 \mathrm{~km}$ for the periodic source with the frequency $\omega=\Omega=2 \pi / T$, where $T$ is the IAR fundamental period.

Dependence of $\left(J_{z} / J_{0}\right)_{\max }$ on the emitted pulse duration is shown in Fig. 3a more evidently. A clear main maximum $\left(J_{z} / J_{0}\right)_{\max }=0.235$ is seen in it which corresponds to the frequency $\omega_{R}=\Omega / 1.5$. At a lower frequency $\omega / \Omega \approx 0.2$ an additional maximum is seen where $\left(J_{z} / J_{0}\right)_{\max }=0.192$. For $\omega / \Omega<0.2$ the amplitude of the trapped waves decreases quickly with decreasing frequency, despite the increase of time integrated energy in the pulse emitted by the source. The frequency of the waves trapped by the resonator is close to $\Omega$. These waves are generated by a pulse from the source, and each of the trapped waves is damped out. If the frequency is $\omega \ll \Omega$, then only the waves generated during the last passage of the emitted pulse through the resonator can be detected as trapped waves separately from this pulse. The characteristic time of this pulse decrease is approximately equal to $1 / \omega$. The trapped waves can be detected separately if this time is smaller than the characteristic time of damping of the trapped waves (see Eq. 14), i.e. if $1 / \omega<2 \pi / \Omega$ or $\omega / \Omega>1 / 2 \pi \approx 0.16$. The low-frequency maximum at $\omega / \Omega \approx 0.2$ corresponds to this condition.

\section{Periodic Alfvén wave}

Let function $F(t)$ in the boundary condition (Eq. 9a) be

$F(t)=\sin (\omega t)$ for $t \geq 0$,

i.e. the source is switched on at time $t=0$ and radiates a periodic Alfvén wave. After some time a periodic process will be established in the resonator region whose amplitude will not vary in time.

The process of a steady-state establishment for $\omega=\Omega$ is shown in Fig. 4. It is seen that it takes $t \approx 8 T$ for the solution to come to a steady-state where $T=2 \pi / \Omega$. But even at $t=4 T$ the amplitude of the waves retained by the resonator is not very different from the maximum one: $\left(J_{z} / J_{0}\right)_{\max }=1.55$, 1.62 and 1.63 at $t=4 T, 8 T$, and $10 T$.

Figure $3 \mathrm{~b}$ shows the amplitude-frequency characteristic of the resonator for the steady-state situation, where $\left(J_{z} / J_{0}\right)_{\max }$ is the greatest value of $J_{z} / J_{0}$ maximum in the height interval 200 to $800 \mathrm{~km}$. It is seen that for a wide range of frequencies $\left(J_{z} / J_{0}\right)_{\max }>1$, i.e. the energy which has come from a periodic source can be stored by the resonator and exceed the losses due to radiation into the magnetosphere. At the amplitude-frequency characteristic of the resonator a number of maxima can be seen. The frequencies corresponding to these maxima are resonant frequencies.

Table 1 gives the resonant frequencies $\omega_{n}$ and $\left(J_{z} / J_{0}\right)_{\max }$ values for the steady-state situation. The approximation of these frequencies for $1 \leq n<10$ is

$\omega_{n}=\Omega\left[(n+0.5) / 1.5-0.009(n-1)^{2}\right]$.

Table 1 shows that the approximation (Eq. 19) is sufficiently exact. Note that the contribution of the nonlinear term in Eq. (19) becomes appreciable only at $\mathrm{n}>5$. Therefore, it can be neglected in most cases of practical interest. In Fig. $3 \mathrm{~b}$, the most clear $\left(J_{z} / J_{0}\right)_{\max }$ maximum is seen at the fundamental resonant frequency $\omega_{1}=\Omega$. As the frequency increases, the $\left(J_{z} / J_{0}\right)_{\max }$ maxima become less distinct. The main part of energy of the waves retained by the resonator is concentrated close to the central part of the resonator in the height interval $h \approx h_{m} \pm 0.25 L$. It is evident from Fig. 5 , that the dependences of $J_{z} / J_{0}$ on height are given for the first four resonant frequencies at fixed points in time when for each of these frequencies the $\left(J_{z} / J_{0}\right)_{\max }$ value becomes maximum at one of these points. In the considered case these maxima are located at the heights $390,340,315$ and $295 \mathrm{~km}$ for the resonant frequencies $\omega_{1}, \omega_{2}, \omega_{3}$, and $\omega_{4}$, correspondingly. 
Table 2. Dependence of the resonator eigenfrequencies on factor $K$ in Eq. (20).

\begin{tabular}{cccccc}
\hline$K$ & $\omega$ & $\omega_{2} / \Omega$ & $\omega_{3} / \Omega$ & $\omega_{4} / \Omega$ & $\omega_{5} / \Omega$ \\
\hline 0.6 & 6.19 & 1.67 & 2.30 & 2.90 & 3.48 \\
0.8 & 6.67 & 1.67 & 2.28 & 2.87 & 3.44 \\
1.0 & 7.02 & 1.67 & 2.27 & 2.86 & 3.44 \\
1.2 & 7.25 & 1.67 & 2.27 & 2.86 & 3.44 \\
1.4 & 7.43 & 1.67 & 2.27 & 2.86 & 3.44 \\
\hline
\end{tabular}

The resonant frequencies $\omega_{n}$ are eigenfrequencies of the resonator. In order to check this conclusion we have in addition solved the problem on eigenvalues when in the initial Eq. (8) the derivative on time is replaced with the complex frequency which was determined from the problem solution. The real values of the resonator eigenfrequencies obtained from this solution differ from the approximation (Eq. 19) by less than $4 \%$ for $n<10$. It implies, in particular, that resonant frequencies will not change if the periodic source is placed in the magnetosphere, under the additional condition that the emitted wave frequency does not change over the wave propagation way from the source to the IAR.

Note that if one sets formally $n=0.5$ in Eq. (19) then the optimum frequency $\omega_{R}$ can be obtained for a single pulse (Eq. 17) when the amplitude of the waves trapped by the resonator is maximum. Nevertheless, the difference between dependences of amplitudes of trapped and retained waves on frequency is essential. For example, Fig. 3 shows that for a periodic source with frequency $\omega=\omega_{R}$ the amplitude of retained waves corresponds to a local minimum $\left(\left(J_{z} / J_{0}\right)_{\max }=0.98\right)$, i.e. the energy of the waves with this frequency is not accumulated by the resonator. For a single pulse of duration $\tau=\pi / \omega_{R}$ the amplitude of the trapped waves is maximum $\left(\left(J_{z} / J_{0}\right)_{\max }=0.235\right)$. In turn, optimum conditions for trapping the waves are at a pulse duration which does not correspond to an eigenfrequency of the resonator, therefore, the amplitude of the waves trapped by the resonator is relatively low even for optimum conditions.

The above estimations were obtained for a specific kind of dependence of the Alfvén wave refractive index $n_{A}$ on height $h$ (see Eq. 10). In a more general case one can assume that in Eq. (10)

$x=4\left(h-h_{m}\right) / L$ for $h \geq h_{m}, \quad x=K 4\left(h-h_{m}\right) / L$ for $h \leq h_{m}$,

where $K$ is a numerical factor for the IAR bottom wall. The interval $0.6 \leq K \leq 1.4$ seems to overlap a possible range of $K$ change for typical daytime and nighttime conditions at middle and high latitudes. Analysis showed that for this range of $K$ change the resonator fundamental frequency $\Omega$ depends on $K$ but $\omega_{n} / \Omega$ values almost do not depend on $K$, at least, for $\mathrm{n} \leq 5$. It is seen more evidently from Table 2 where results of the solution of the problem on eigenvalues are shown for the case when for the interval $h_{0} \leq h \leq h_{m}$ the approximation (Eq. 10) is used in which $x$ is described by Eq. (20). For the

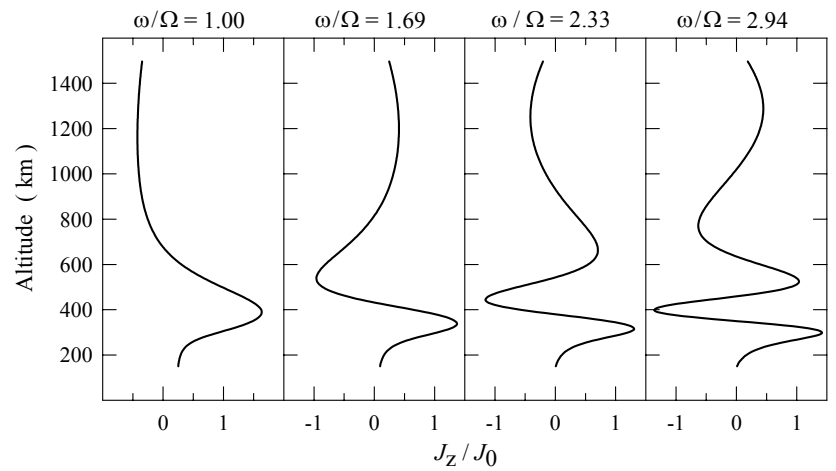

Fig. 5. Altitudinal distributions of relative values of the fieldaligned current $J_{z} / J_{0}$ for four resonant frequencies at fixed points in time $t$ which correspond to $J_{z} / J_{0}$ maximum in the IAR.

interval $h_{m} \leq h \leq h_{1}$ the IRI model is used as before. Table 2 shows that in the interval $0.6 \leq K \leq 1.4$ the fundamental frequency $\Omega$ changes approximately by $20 \%$ and changes in the resonant frequencies $\omega_{n} / \Omega$ do not exceed $2 \%$. The difference between $\omega_{n} / \Omega$ values given in Table 2 and the approximation (Eq. 19) does not exceed 2-3\%. It implies that Eq. (19) for $n \leq 5$ is almost universal, i.e. it is applicable for a wide range of geophysical conditions, including day hours. More detailed analysis of this property of IAR is beyond the scope of this paper.

\section{Discussion}

The above stated dependences of $\Omega$ and $Q$ on $n_{A}$ altitude distribution were obtained on the basis of the analytical approximation of the problem numerical solution. Similar dependences were obtained on the basis of analytical solution of the problem on eigenvalues with simplifying assumptions of $n_{A}$ distribution (Polyakov and Rapoport, 1981; Lysak, 1991, 1999). We compared both results using the above notations and neglecting energy losses of the waves in the E layer. For the version when $\sin I=1$,

$\begin{array}{ll}n_{A}^{2}=n_{A m}^{2}\left(\varepsilon^{2}+\exp \left(-2\left(h-h_{m}\right) / L\right),\right. & h \geq h_{m} \\ n_{A}^{2}=n_{A m}^{2}, & h_{m}>h \geq h_{0}\end{array}$

and $\varepsilon \omega L / V_{A m} \ll 1$ it was obtained (Polyakov and Rapoport, 1981) that the fundamental frequency and Q-factor of the resonator on this frequency are

$\Omega=1.25 \pi V_{A m} /(L+\Delta h), Q=(1+\Delta h / L) /(\pi \varepsilon)$,

where $\Delta h=h_{m}-h_{0}$. For the above values of $h_{m}=345 \mathrm{~km}$, $h_{0}=150 \mathrm{~km}$, and $L=240 \mathrm{~km}$ used we obtain $\Delta h / L=0.81$, $\Omega=2.2 V_{A m} / L$, and $Q=0.6 / \varepsilon$. It is seen that the analytical results (Eq. 22) are not strongly different from the numerical ones (Eq. 14) and (Eq. 16). Nevertheless, the altitude distribution (Eq. 21) apparently occurs seldom. In order to approach a realistic situation, another sense should be given to $\Delta h$ in Eq. (22) - that it is the characteristic altitudinal scale of change of the Alfvén wave refractive index at the resonator 
bottom wall. As obtained above for the $n_{A}$ altitudinal distribution in the form (Eq. 10), its characteristic scale at the resonator bottom wall is approximately equal to $0.5 \mathrm{~L}$, i.e. $\Delta h / L=0.5$. Substitution of this $\Delta h$ value into Eq. (22) gives $\Omega=2.6 V_{A m} / L, Q=0.5 / \varepsilon$, which coincides with Eqs. (14) and (16) at $\sin I=1$.

For a simpler version when $\sin I=1$, the $n_{A}$ altitude distribution is described by Eq. (21) with $h \geq h_{m}$, the bottom boundary condition is specified at the height $h=h_{m}$, and $\varepsilon \omega L / V_{A m} \ll 1$; Lysak (1991) obtained that eigenfrequencies of the resonator are determined by the condition $J_{0}\left(\omega L / V_{A m}\right)=0$, where $J_{0}$ is Bessel function. Recall that the first five zeroes of $J_{0}$ are 2.40, 5.52, 8.65, 11.79, and 14.93. Hence, the resonator fundamental frequency $\Omega$ is equal to $2.4 V_{A m} / L$ (Lysak, 1991), and

$\omega_{n} \approx \Omega[1.305 n-0.305], \Delta \omega=\omega(n+1)-\omega(n) \approx 1.305 \Omega$

One can see that the fundamental frequency $\Omega$ differs weakly from Eq. (14) at $\sin I=1$, but the interval $\Delta \omega$ is approximately twice the interval resulted from Eq. (19): $\Delta \omega \approx 0.67 \Omega$. As an illustrative example, Eq. (19) is written for $n=1,3,5$, i.e. for the first odd values of $n$ :

$\omega_{2 n-1} \approx \Omega[(2 n-1)+0.5] / 1.5 \approx \Omega(1.33 n-0.33)$.

The odd eigenfrequencies in Eq. (19) are seen to be very close to those obtained by Lysak (1991). It was noted above that the scale of the resonator bottom wall is approximately equal to $0.5 \mathrm{~L}$. Therefore, simultaneous account of the upper and lower parts of the resonator results in the occurrence of additional eigenfrequencies which correspond to even values of $n$ in Eq. (19).

We did not take into account Hall currents, therefore we cannot compare our results with ground-based observations. Nevertheless, we can present here some preliminary estimations. For daytime conditions at high latitudes on the basis of the solution of the general problem for a periodic source, Lysak (1999) has obtained that in addition to the fundamental frequency $F=f_{1}=0.2 \mathrm{~Hz}$ there are two additional maxima: $f_{2}=0.33 \mathrm{~Hz}$ and $f_{3}=0.5 \mathrm{~Hz}$. Equation (19) can be rewritten as $f_{n} \approx F(n+0.5) / 1.5$. From this equation it follows that $f_{2}=0.33 \mathrm{~Hz}$ and $f_{3}=0.47 \mathrm{~Hz}$, if $F=0.2 \mathrm{~Hz}$. It is seen that the frequencies obtained by Lysak coincide almost exactly with the approximation (Eq. 19). The ground effect of these waves has appeared essentially different: amplitudes of the waves $f_{1}$ and $f_{3}$ are approximately 3 times as $f_{2}$ amplitude (Lysak, 1999). Therefore, on data of observations in the region of the resonator the interval $\Delta f$ will be smaller than on the groundbased data (Lysak, 1999).

A similar conclusion can be deduced from data of ground-based observations of the spectral resonance structure (SRS) at middle latitudes (L-shell is approximately equal to 2.65) in winter close to midnight at solar minimum. For these conditions the F2-layer critical frequency foF $2 \approx 2.5 \mathrm{MHz} ; \Delta f=\Delta \omega / 2 \pi \approx 2 \mathrm{~Hz}$ is the typical value but sometimes $\Delta f \approx 1 \mathrm{~Hz}$ (Belyaev et al., 1990). This gives $V_{A m} \approx 870 \mathrm{~km} / \mathrm{s}, \sin I \approx 0.9$. For these conditions $L \approx 210 \mathrm{~km}$, if the estimations are based on the IRI model (Bilitza, 1997). Substitution of these values into Eqs. (14) and (19) gives the fundamental frequency $F=\Omega / 2 \pi \approx 1.5 \mathrm{~Hz}$ and $\Delta f \approx F / 1.5 \approx 1 \mathrm{~Hz}$. The obtained value is seen to coincide with the minimum $\Delta f$ interval of observed ones and is approximately half the typical value of $\Delta f \approx 2 \mathrm{~Hz}$. Hence, in accordance with Lysak's (1999) conclusions, ground-based observations show that the even eigenfrequencies of the IAR for the shear-mode Alfvén waves $\left(f_{2}, f_{4}, \ldots\right)$ are suppressed and distinguished less clearly than the odd eigenfrequencies. These results show that taking into account the isotropic fast compressional waves is important for analysis of the groundbased data. At the same time the results of analysis by neglecting of these waves presented in this paper have allowed us to obtain explicit dependences of characteristics of the shear-mode Alfvén waves on the distribution of the Alfvén wave refractive index along the geomagnetic field, and thus have enabled us to compare illustrative data obtained by a number of different ways and under different geophysical conditions.

\section{Conclusions}

A numerical solution of the problem on the dynamics of Alfvén waves in the IAR region at middle latitudes at nighttime is presented for a case when a source emits a single pulse of Alfvén waves of duration $\tau$ into the resonator region. It was obtained that a part of the pulse energy is trapped by the resonator, i.e. damped Alfvén waves trapped by the resonator are formed. It was found that the amplitude of the trapped waves depends essentially on $\tau$ and it is maximum at $\tau=(3 / 4) T$, where $T$ is a fundamental eigenperiod of the resonator. The maximum amplitude of these waves does not exceed $30 \%$ of the initial pulse even under optimum conditions. Relatively low efficiency of trapping Alfvén waves is caused by the difference between the optimum duration of the pulse and the resonator fundamental period. The period of oscillations of the trapped waves is approximately equal to $T$, irrespective of the pulse duration $\tau$. The characteristic time of damping of the trapped waves is proportional to $T$, therefore the resonator Q-factor for such waves does not depend on $T$.

The situation changes dramatically when a source emits periodic Alfvén waves. The amplitude of the waves coming from such a source can be increased in the resonator region by more than $50 \%$. This implies that the resonator can accumulate and retain the energy of the Alfvén waves, despite energy losses due to radiation into the magnetosphere. The amplitude of the waves in the IAR is maximum on the resonator eigenfrequencies $\omega_{n}$ and this effect is most pronounced when the frequency of the source coincides with the resonator fundamental frequency $\omega_{1}=\Omega=2 \pi / T$. The interval between the first adjacent eigenfrequencies of the resonator is $\Delta \omega \approx \Omega / 1.5$.

For a single pulse the effective frequency $\omega=\pi / \tau=\Omega / 1.5$ is optimum. For a periodic source the dependence of am- 
plitude of the retained waves on frequency $\omega$ is such that $\omega=\Omega / 1.5$ corresponds to a local minimum and the waves of such a frequency do not accumulate energy in the resonator region. This alone is the basic difference between efficiencies of pulse and periodic sources of the Alfvén waves.

Explicit dependences of the resonator fundamental frequency $\Omega$, harmonics of this frequency $\omega_{n}$, characteristic time of damping Alfvén waves trapped by the resonator, and the resonator Q-factor for these waves on altitude distribution of the Alfvén wave refractive index were obtained. These dependences are analytical approximations of the numerical solution of this problem. These dependences were compared with the ones obtained previously by other methods.

Acknowledgements. This work was supported by the Russian Foundation for Basic Research, project No. 01-05-03890-p98, and International Science and Technology Center, project No. 1328/99.

Topical Editor T. Pulkkinen thanks a referee for his/her help in evaluating this paper.

\section{References}

Belyaev, P. P., Polyakov, S. V., Rapoport, V. O., and Trakhtengerts, V. Yu.: The ionospheric Alfvén resonator, J. Atmos. Terr. Phys., 52, 781-788, 1990.

Belyaev, P. P., Bosinger, T., Isaev, S. V., and Kangas, J.: First evidence at high latitudes for the ionospheric Alfvén resonator, J. Geophys. Res, 104, 4305-4317, 1999.

Belyaev, P. P., Polyakov, S. V., Ermakov, E. N., and Isaev, S. V.: Solar cycle variations in the ionospheric Alfvén resonator 19851995, J. Atmos. Solar-Terr. Phys., 62, 239-248, 2000.

Bilitza, D.: International Reference Ionosphere - Status 1995/96, Adv. Space Res., 20, 1751-1754, 1997.

Carpenter, D. L. and Anderson, R. R.: An ISEE/Whistler model of equatorial electron density in the magnetosphere, J. Geophys. Res., 97, 1097-1108, 1992.

Demekhov, A. G., Belyaev, P. P., Isaev, S. V., Manninen, J., Turunen, T., and Kangas, J.: Modelling the diurnal evolution of the resonance spectral structure of the atmospheric noise background in the Pc1 frequency range, J. Atmos. Solar-Terr. Phys., 62, 257-265, 2000.

Deminov, M. G., Oraevsky, V. N., and Ruzhin, Yu. Ya.: Ionospheremagnetosphere effects of rockets launched toward high latitudes, Geomagn. Aeron., 41, 738-745, 2001.
Gaidukov, V. M., Deminov, M. G., Dumin, Yu. V., Omelchenko, A. N., Romanovsky, Yu. A., and Feigin, V. M.: "Auroral trigger" experiment, 1. Generation of electric fields and particle fluxes by plasma injection into the ionosphere at high latitudes, Kosmicheskie Issledovaniya (in Russian), 31, 54-62, 1993.

Grzesiak, M.: Ionospheric Alfvén resonator as seen by Freja satellite, Geophys. Res. Lett., 27, 923-925, 2000.

Lysak, R. L.: Coupling of the dynamic ionosphere to auroral flux tubes, J. Geophys. Res., 91, 7047-7056, 1986.

Lysak, R. L.: Theory of auroral zone PiB pulsation spectra, J. Geophys. Res., 93, 5942-5946, 1988.

Lysak, R. L.: Feedback instability of the ionospheric resonant cavity, J Geophys. Res., 96, 1553-1568, 1991.

Lysak, R. L.: Propagation of Alfvén waves through the ionosphere, Phys. Chem. Earth., 22, 757-766, 1997.

Lysak, R. L.: Propagation of Alfvén waves through the ionosphere: Dependence on ionospheric parameters, J. Geophys. Res., 104, 10 017-10 030, 1999.

Marklund, G., Brenning, N., Holmgren, G., and Haerendelm G.: On transient electric fields observed in chemical release experiments by rockets, J. Geophys. Res., 92, 4590-4600, 1987.

Nishida, A.: Geomagnetic diagnosis of the magnetosphere, Springer-Verlag, N.Y., 1978.

Pokhotelov, O. A., Pokhotelov, D., Streltsov, A., Khruschev, V., and Parrot, M.: Dispersive ionospheric resonator, J. Geophys. Res., 105, 7737-7746, 2000.

Pokhotelov, O. A., Khruschev, V., Parrot, M., Senchenkov, S., and Pavlenko, V. P.: Ionospheric Alfvén resonator revisited: Feedback instability, J. Geophys. Res., 106, 25 813-25 824, 2001.

Polyakov, S. V. and Rapoport, V. O.: The ionospheric Alfvén resonator, Geomagn. Aeron., 21, 610-614, 1981.

Prikner, K., Mursula, K., Feygin, F. Z., Kangas, J., Kerttula, R., Pikkarainen, T., Pokhotelov, O. A., and Vagner, V.: Nonstationary Alfvén resonator: vertical profiles of wave characteristics, J. Atmos. Solar-Terr. Phys., 62, 311-322, 2000.

Rozhdestvensky, B. L. and Yanenko, N. N.: Systems of quasilinear equations and their application to gas dynamics (in Russian), Nauka Publishing, Moscow, 1978.

Trakhtengertz, V. Y. and Feldstein, A. Y.: Quiet auroral arcs: Ionospheric effect of magnetospheric convection stratification, Planet. Space Sci., 32, 127-134, 1984.

Trakhtengertz, V. Y. and Feldstein A. Y.: Turbulent Alfvén boundary layer in the polar ionosphere 1, Excitation conditions and energetics, J. Geophys. Res., 96, 19363-19474, 1991. 